Textures and Microstructures, 1991, Vols 14-18, pp. 1173-1178 Reprints available from the publisher

Photocopying permitted by license only
(C) 1991 Gordon and Breach Science Publishers SA Printed in the United Kingdom

\title{
ANISOTROPIC PLASTIC BEHAVIOR ASSOCIATED WITH ROLLING TEXTURES
}

\author{
M.DARRIEULAT ard F.MONTHEILLET \\ Ecol€ des Mines, Département Matériaux, 158 cours Fauriel, \\ 42023 Saint-Etienne Cedex 2, France.
}

\section{ABSTRACT}

The overall plastic response of a sheet can be simply calculated from the knowledge of its various texture components, the behavior of them being described by the equations of continuum plasticity. It is shown, however, that the classical assumptions used for combining the elementary components can lead to significantly different predictions of the sheet behavior.

\section{INTRODUCTION}

Texture components in rolled sheet of cubic metals either are self-symmetric (e.g. the cube $\{001\}<100\rangle$ or the Goss $\{110\}<001>$ orientation) or must be combined into twinsymmetric (e.g. the $\mathrm{Cu}\{112\}<11 \overline{1}>$ or the Bs $\{110\}<1 \overline{1} 2>$ orientation) or four-fold-symmetric (e.g. the $S\{123\}<63 \frac{4}{4}>$ orientation) 'rolling texture components' in order to neet the orthotropic symmetry of the material. In the CMTP method (Continuum Mechanics of Textured Polycrystals) first proposed by Montheillet et al. ${ }^{1}$ and then applied to rolled sheet by Lequeu et al. ${ }^{2-6}$, the material is considered as a set of texture components, the behavior of which is described by the equations of continuum plasticity. One of the main problems raised by the theory is the combination of the texture components into rolling components, as well as the averaging procedure required when several crystallographic types of texture components are present. The aim of this paper is to compare the two classical assumptions which can be used for such combinations and to assess their influence on the overall orthotropic plastic response of the sheet.

\section{PLASTIC BEHAVIOR OF A SINGLE TEXTURE COMPONENT}

Any texture component consists in a distribution of grains more or less disoriented with respect to the ideal orientation \{hk1\}<uvw>. If the scatter width is the same along any direction of angular space, the associated yield surface exhibits cubic symmetry with respect to the $\langle 100\rangle$ axes of the ideal orientation. It can thus be described by the Hill quadratic yield equation, in which the additional condition of cubic symmetry is obtained by prescribing that 
the three $\langle 100\rangle$ axes be equivalent. The flow rule is then derived from the normality principle and can be written in the 6-dimensional vector form ${ }^{7}$ :

$$
\dot{\vec{\epsilon}}=(3 \dot{\bar{\epsilon}} / 2 \bar{\sigma})\{\mathrm{A}[\mathrm{U}]+(1-\mathrm{A})[\mathrm{V}]\} \overrightarrow{\mathbf{s}}
$$

where $\dot{\bar{\epsilon}}$ is an equivalent strain rate and $\bar{\sigma}$ an equivalent stress defined such that the rate of plastic work per unit volume $\dot{w}=\bar{\sigma} \bar{\epsilon}$. The reciprocal of eq.(1), or constitutive equation, takes the form ${ }^{7}$ :

$$
\overrightarrow{\mathbf{s}}=(2 \bar{\sigma} / 3 \dot{\bar{\epsilon}})\{(1 / \mathrm{A})[\mathrm{U}]+(1-1 / \mathrm{A})[\mathrm{V}]\} \dot{\overrightarrow{\boldsymbol{\epsilon}}}
$$

In the above equations, $\bar{\sigma}$ is generally considered as a material constant $\sigma_{0}$ which determines the size of the yield surface, whereas the anisotropy coefficient A is associated with the shape of the latter. In the case of fcc or bcc metals deforming by crystallographic slip, A has been related to the shape of the critical polyhedron ${ }^{1,8}$. A value of $A=0.409$ corresponding to the $\{111\}<110\rangle$ slip systems will be used here. It is also possible to consider a dual yield surface in the strain rate space ${ }^{9}$. In such a case, $\dot{\bar{\epsilon}}=\dot{\epsilon}_{0}$ is a material constant in place of $\bar{\sigma}$. Finaily, [U] is the identity matrix and [V] is a $6 \times 6$ matrix, the elements of which depend on the Miller indices $h, k, 1$ and $u, v, w$ of the ideal orientation?

\section{ASSUMPTIONS USED TO COMBINE THE TEXTURE COMPONENTS}

Two classical hypotheses are considered here :

(i) the uniform strain rate (Taylor) assumption : $\dot{\boldsymbol{\epsilon}}^{\mathbf{i}}=\dot{\boldsymbol{\epsilon}}$ for any component $i$; (ii) the uniform stress (stâtic) assumption : $\mathbf{s}^{\mathbf{i}}=\mathbf{s}$ for any component $\mathbf{i}$.

It should be noted that the first one does not allow the strain rate yield criterion to be satisfied in every texture component, but only for the whole material ; the same remark holds for the second assumption when a stress yield function is used. Furthermore, when the Taylor or the static assumption is used, the equivalent strain rate $\dot{\bar{\epsilon}}^{i}$ or stress $\bar{\sigma}^{i}$ takes different values in the various components, respectively. It can be shown, however, that the differences remain rather small ${ }^{10}$, such that, to a first approximation, $\dot{\epsilon}^{i}$ or $\bar{\sigma}^{i}$ can be identified to the overall equivalent strain rate $\dot{\bar{\epsilon}}$ or stress $\bar{\sigma}$.

The plastic response of the whole sheet is then written in the form (uniform stress) :

$$
\dot{\overrightarrow{\boldsymbol{\epsilon}}}=(3 \dot{\bar{\epsilon}} / 2 \bar{\sigma})\left\{\left(\mathrm{f}^{0}+\mathrm{A} \Sigma \mathrm{f}^{\mathrm{i}}\right)[\mathrm{U}]+(1-\mathrm{A}) \Sigma \mathrm{f}^{\mathrm{i}}\left[\mathrm{V}^{\mathrm{i}}\right]\right\} \overrightarrow{\mathbf{s}}
$$

or in the reciprocal form (uniform strain rate) :

$\overrightarrow{\mathbf{s}}=(2 \bar{\sigma} / 3 \dot{\bar{\epsilon}})\left\{\left(\mathrm{f}^{0}+(1 / \mathrm{A}) \Sigma \mathrm{f}^{\mathrm{i}}\right)[\mathrm{U}]+(1-1 / \mathrm{A}) \Sigma \mathrm{f}^{\mathrm{i}}\left[\mathrm{V}^{\mathrm{i}}\right]\right\} \dot{\overrightarrow{\boldsymbol{\epsilon}}}(4)$

where the volume fractions $f^{i}$ and the orientation dependent matrices $\left[\mathrm{V}^{\mathrm{i}}\right]$ are associated with the texture component $i$ and the volume fraction $f^{0}$ with the 'isotropic component' of the material. Finally, $\bar{\sigma}=\sigma_{0}$ or $\dot{\bar{\epsilon}}=\dot{\epsilon}_{0}$ according to the type of yield function considered. 


\section{RESULTS}

To assess the influence of the above assumptions on the predicted plastic behavior of a sheet, tension tests were calculated. In this section, the specimen axes will be referred to as 1 (tensile direction), 2 (width direction) and 3 (thickness direction). Two sets of boundary conditions can be prescribed alternatively : on the one hand, uniaxial tension is associated with long and narrow samples, which means that all stresses are zero except the tension component $\sigma_{11}$. From symmetry considerations, the only non zero shear strain rate is the in-plane component $\dot{\epsilon}_{12}$. On the other hand, 'orthogonal' (or shear strain free) tension is likely to occur when short and wide specimens are used. All shear strain rates then vanish and the only non zero shear stress is the $\sigma_{12}$ component. Boundary conditions are difficult to control in real tests, but are nevertheless expected to lie between these two extremes.

Calculations were first carried out for single rolling texture components. The plastic behavior associated with twin or four-fold-symmetric components is sensitive to the assumption used to combine the two or four elementary texture components, respectively. Results obtained for the Brass $\{110\}<112>$ rolling component are depicted in Fig. 1 in the case of uniaxial tension. Fig.la first shows the angular variations of the normalized tension stress $\sigma / \sigma_{0}$. Here a classical stress yield function was used and the tension strain rate was prescribed. If, alternatively, a strain rate yield surface is assumed, then the tension stress $\sigma$ is prescribed. Fig.la also shows the $\theta$ dependence of the corresponding normalized tension strain rate $\dot{\epsilon} / \dot{\epsilon}_{0}$. It should be noted that the static hypothesis leads to lower values of the tension stress in the first case and accordingly larger values of the tension strain rate in the second. This is in agreement with the common statement that the uniform strain rate and uniform stress assumptions provide upper and lower bounds, respectively, for the rate of plastic work $\dot{w}=\sigma \dot{\epsilon}$ in the case of perfectly plastic materials obeying a stress yield function. The $\theta$ dependence of the strain rate ratio $\mathrm{R}=\dot{\epsilon}_{22} / \dot{\epsilon}_{33}$ and the normalized shear strain rate $\dot{\epsilon}_{12} / \dot{\epsilon}_{11}$ are shown in Figs $1 \mathrm{~b}$ and c. The results are the same for stress and strain rate yield surfaces, but are rather sensitive to the combination hypothesis. The same calculations carried out on other rolling texture components bring into evidence that $K$ is generally more influenced than $\sigma$ (or $\dot{\epsilon}$ ) by the assumption used for combining the elementary components.

Another example is given by a sheet exhibiting only the (self-symmetric) Goss and the isotropic random components (the behavior of the latter is described by eqs (1) and (2) when $A=1)$. The value of $R(\pi / 2)$ is plotted in Fig. 2 as a function of the fraction of Goss component. It can be seen that the discrepancy introduced by the combination hypotheses can reach $50 \%$ for a Goss fraction of about: 0.7 . 
Finally, Figs $3 a$ and $b$ display results for a sheet exhibiting rolling components of several crystallographic types, together with experimental data from the literaturel The texture was analyzed as a mixture of $57 \%$ Bs $\{110\}<1 \overline{1} 2>, 29 \%$ Goss $\{110\}<001>$ and $14 \%\{311\}<112>$ rolling texture components. Here, all the theoretical curves fall into a scatter band delimited by the Taylor uniaxial and static orthogonal tests. Fig.3a shows that the tension stress is rather well predicted, whatever the assumption used. The agreement with the data is not so good for the strain rate ratio (Fig.3b), although the general trend is well reproduced. This could be due to the too 'rounded' quadratic yield surface which was used here to describe the plastic behavior of the texture components. However, $R$ calculations carried out for other mixtures of rolling components show that discrepancies of up to $33 \%$ are commonly introduced by the choice of either the boundary conditions (uniaxial vs. orthogonal) or the combination hypothesis (uniform strain vs. uniform stress).

\section{CONCLUSIONS}

The few results presented above as examples show that (i) The assumptions of uniform strain or uniform stress used for combining the various texture components present in a sheet can lead to significantly different predictions of the plastic behavior.

(ii) The largest discrepancies occur for the theoretical strain rate ratios and in-plane shear strains, where they are likely to exceed the experimental uncertainties.

(iii) The calculated tension stresses and strain rate ratios are also influenced by the uniaxial or shear free boundary conditions assumed for the tension test.

(iv) However, progress is still necessary to improve the CMTP method, in particular by a better description of the yield surface associated with each texture component.

\section{REFERENCES}

1. F.Montheillet, P.Gilormini and J.J.Jonas, Acta Metall., 33,705 (1985).

2. Ph.Lequeu, F.Montheillet and J.J.Jonas, in Textures in Non-Ferrous Metals and Alloys (The Metallurgical Society, Warrendale PA, 1985), p.189.

3. Ph.Lequeu, Ph.D. Thesis, McGill Univ., Montreal (1986).

4. Ph.Lequeu, P.Gilormini, F.Montheiliet, B.Bacroix and J.J.Jonas, Acta Metall., 35, 1159 (1987).

5. Ph.Lequeu and J.J.Jonas, Metal1. Trans., 19A, 105 (1988).

6. F.Montheillet, Ph.Lequeu, P.Gilormini and J.J.Jonas, Proc. ICOTOM 8, p.1031 (1988).

7. M.Darrieulat, R.Fortunier and F.Montheillet, to be published.

8. F.Montheillet, B.Orlans-Joliet and Ph.Tavernier, Matér. Tech. (Paris), 35 (sept.-oct. 1989).

9. R.Fortunier, J. Mech. Phys. Solids, 37, 779 (1989).

10. F.Montheiliet and M.Darrieulat, submitted to ICM6.

11. A.W. Stephens, Ph.D. Thesis, Univ. of Arizona (1968). 


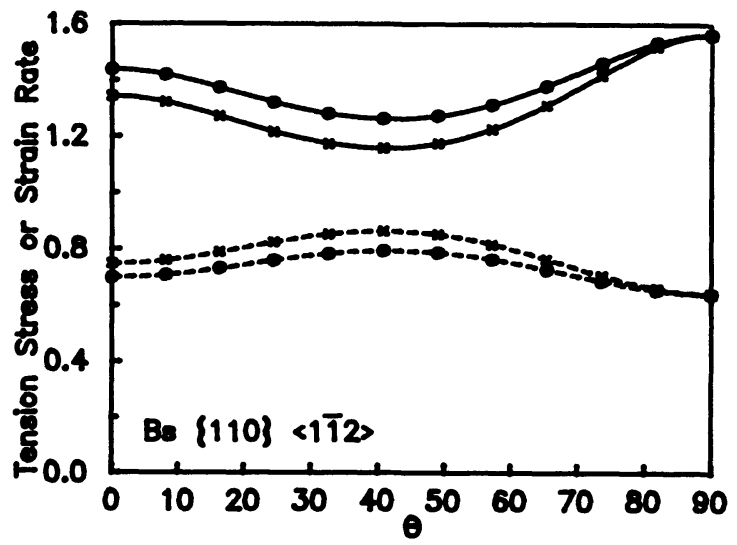

a

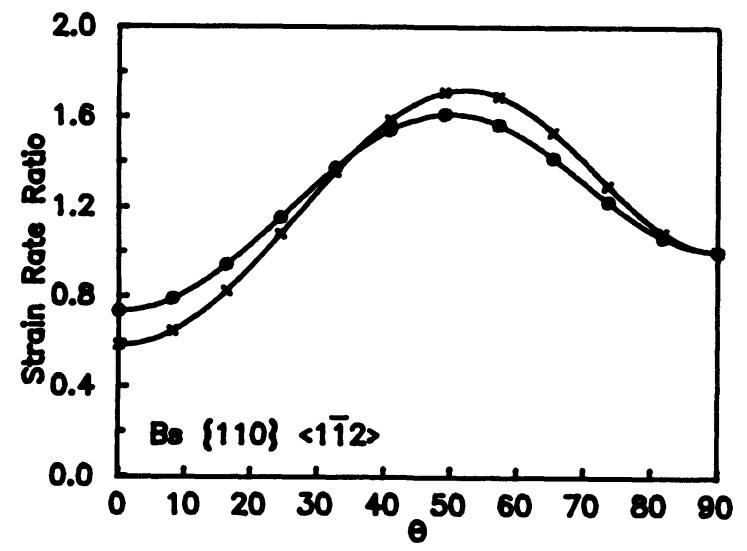

b

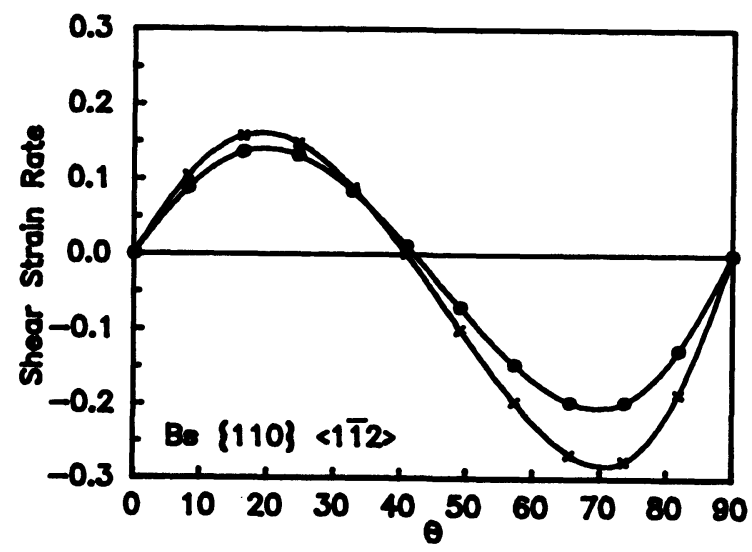

C

Figure 1. Angular variations of the normalized tension stress (full lines) and strain rate (broken lines) (a), the strain rate ratio (b) and the normalized shear strain rate (c) for the Brass rolling texture component. Comparison of the Taylor $(\bullet)$ and the static ( $x$ ) assumptions. 


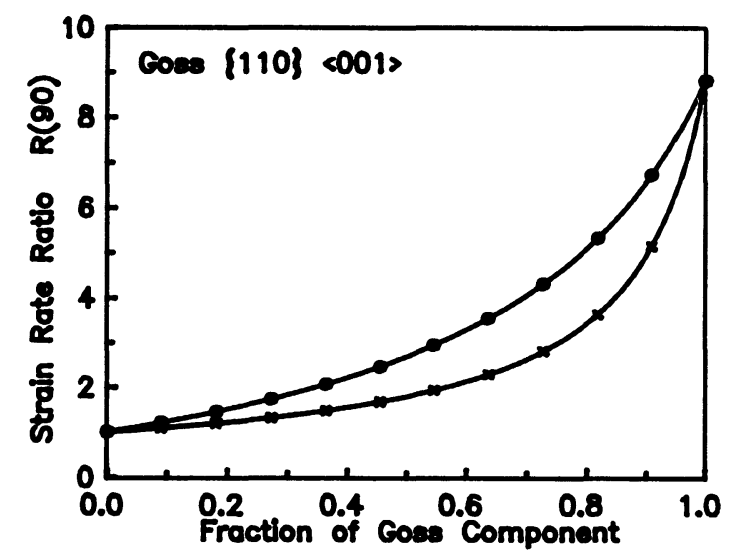

Figure 2. Strain rate ratio $R(\pi / 2)$ along the transverse direction of a sheet exhibiting various fractions of the Goss texture component. Comparison of the Taylor $(\bullet)$ and the static $(\mathrm{x})$ assumptions.

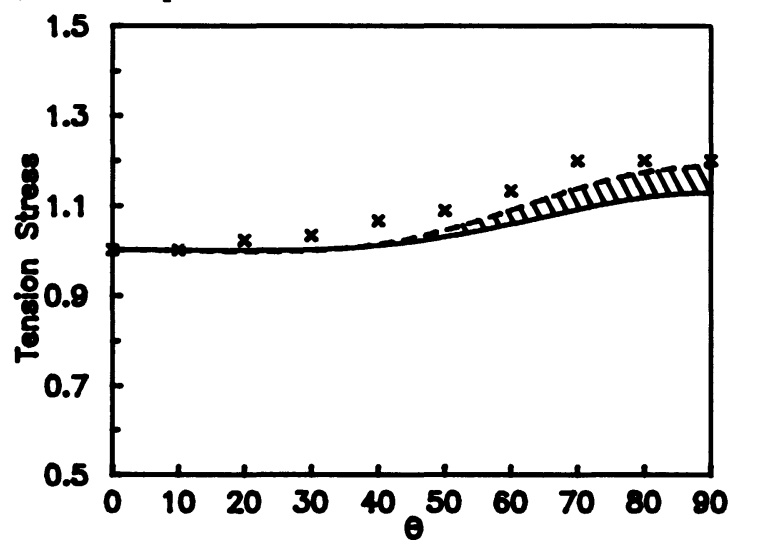

a

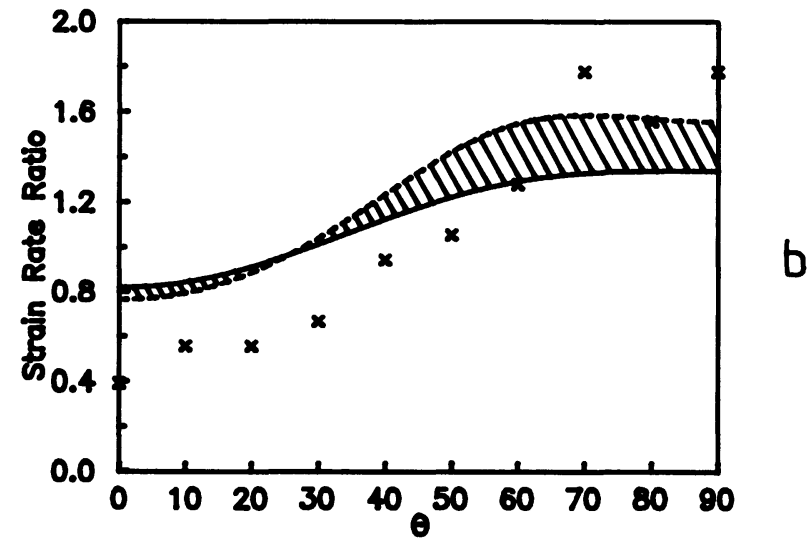

Figure 3. Angular variations of the normalized tension stress (a) and the strain rate ratio (b) for a brass sheet with several rolling texture components (see text). The Taylor uniaxial (full lines) and static orthogonal (broken lines) assumptions are compared with experimental data $(x)$. 